\title{
Reframing Time Spent Alone: Reappraisal Buffers the Emotional Effects of Isolation
}

\author{
Micaela Rodriguez ${ }^{1,2} \odot \cdot$ Benjamin W. Bellet ${ }^{1} \cdot$ Richard J. McNally $^{1}$
}

Published online: 4 July 2020

(c) Springer Science+Business Media, LLC, part of Springer Nature 2020

\begin{abstract}
Background Loneliness, a transdiagnostic feature of psychopathology, is an experience of perceived isolation only weakly linked to the amount of time spent alone. Although traditional loneliness interventions aim to increase social contact, targeting maladaptive cognition about time alone may be an effective way to reduce loneliness. We investigated whether a brief reappraisal manipulation enables individuals to experience their time alone more positively. We also tested the impact of trait loneliness, compulsive social media use, and trait reappraisal on experiences of time alone.

Methods College students and community members $(N=220)$ were randomly assigned to read a passage about the benefits of solitude $(n=74)$, the true prevalence of loneliness $(n=72)$, or a control topic $(n=74)$. Participants then sat alone for $10 \mathrm{~min}$. Results Across conditions, positive and negative mood significantly decreased after sitting alone. Participants who read about the benefits of solitude experienced a smaller reduction in positive mood than those in the control condition. Participants who less frequently used reappraisal in their everyday lives benefited most from the manipulation.

Conclusions Our results provide preliminary evidence that reappraising time alone as solitude may boost resilience to the decrements in positive mood associated with time alone. Limitations, clinical implications, and directions for future research are discussed.
\end{abstract}

Keywords Loneliness $\cdot$ Solitude $\cdot$ Reappraisal $\cdot$ Alone time $\cdot$ Emotion

\section{Introduction}

Again and again, U.S media headlines warn us of a "loneliness epidemic" that is plaguing our nation (Brooks 2018). Self-reported loneliness levels are higher today than they have ever been (Jeste et al. 2020): Half of Americans either sometimes or always feel lonely, and $25 \%$ experience chronic loneliness (Cigna 2018; Hawkley and Cacioppo 2010). Further, social distancing measures enacted in response to the Coronavirus Disease 2019 (COVID-19) pandemic have amplified concerns about loneliness even further in the

Micaela Rodriguez

micaela.rodriguez@nyspi.columbia.edu

1 Department of Psychology, Harvard University, Cambridge, MA, USA

2 Present Address: New York State Psychiatric Institute and Department of Psychiatry, Columbia University College of Physicians and Surgeons, 1051 Riverside Drive, New York 10032, NY, USA population at large (Banerjee and Rai 2020; Fiorillo and Gorwood 2020).

As robust evidence shows, the adverse effects of loneliness on our health are alarming. Loneliness is a transdiagnostic psychiatric risk factor linked to elevated depression severity (Cacioppo et al. 2006), suicidal ideation (Stravynski and Boyer 2001), and social anxiety (Lim et al. 2016). Loneliness has been found to accelerate cognitive decline (Tilvis et al. 2004), elevate blood pressure (Hawkley et al. 2006), and increase risk of Alzheimer's and cardiovascular disease (Wilson et al. 2007). Loneliness has even been linked to premature mortality, with health effects comparable to those of obesity or smoking fifteen cigarettes a day (Holt-Lunstad 2017). Given its far-reaching consequences, loneliness has been deemed a public health crisis by psychologists, medical professionals, and politicians alike (Luo et al. 2012). As many researchers believe COVID-19 will only exacerbate the global loneliness epidemic (Maheshwari and Bronsther 2020), developing effective strategies to reduce loneliness is imperative. 


\section{Two Approaches to Treating Loneliness}

Given its clinical implications, loneliness has been identified as a target of clinical intervention in its own right (Bessaha et al. 2020; Heinrich and Gullone 2006). Existing interventions for loneliness typically target one of two groups of factors that play a role in its development and maintenance: (1) Social (or external) factors and (2) cognitive (or internal) factors (Cornwell and Waite 2009).

\section{Social Approach}

To date, psychological research has focused almost exclusively on the external social factors related to loneliness (Bessaha et al. 2020; Masi et al. 2011). As a result, the majority of loneliness interventions boost social skills, expand opportunities for social interaction, and motivate individuals to create meaningful connections within their communities (Cacioppo et al. 2015; Dill and Anderson 1999). According to a meta-analysis, such interventions have found moderate success in reducing loneliness (Masi et al. 2011); an objective deficit in opportunities for social interactions undoubtedly contributes to perceptions of isolation.

However, it is possible that these social interventions do not adequately address the subjective nature of loneliness (Heinrich and Gullone 2006). By emphasizing the need to increase social contact, these interventions may perpetuate the idea that decreasing the amount of time spent alone is an effective way to conquer loneliness, or that being alone is inherently pathological, increasing the tendency to appraise periods of isolation as loneliness. That is, these interventions may fail to consider that loneliness has little to do with our objective number of social interactions, and more to do with how we perceive ourselves and our social world (Cornwell and Waite 2009).

\section{Cognitive Approach}

Cognitive approaches address the subjective nature of loneliness that social approaches may fail to consider. From a cognitive perspective, loneliness arises from maladaptive social cognition, i.e., negative beliefs and thoughts about ourselves, others, and the world around us. Cognitive interventions for loneliness aim to target these types of social cognition (Masi et al. 2011). According to a metaanalysis of fifty loneliness treatments, interventions that include a cognitive component (e.g. cognitive-behavioral therapy) are significantly more effective in reducing loneliness than are social interventions (Masi et al. 2011).
Nonetheless, cognitive approaches to loneliness have been significantly understudied to date (Cacioppo et al. 2015).

\section{The Role of Time Spent Alone}

Although scientists agree that loneliness impairs our health, we must clearly identify what loneliness really is-and is not. Loneliness is not synonymous with physical isolationin fact, the quantity of social relationships and amount of time we spend alone are very weak predictors of experienced loneliness (Cacioppo et al. 2015; Holt-Lunstad 2017). Rather, loneliness is a subjective state of perceived social isolation. Thus, it may be this perception that predicts adverse health effects, rather than simply our objective isolation [how much time we actually spend alone, or how many friends we have; Cornwell and Waite (2009); Heinrich and Gullone (2006)]. Indeed, one study showed that although self-reported loneliness increased risk of dementia in older adults, how much time individuals spent alone had no effect in the development of the disorder (Holwerda et al. 2014). In another study, lonely adults suffered from more severe physical disease and greater immunological deficiencies than their nonlonely counterparts independent of the size of their social networks (Boss et al. 2015).

Spending time alone does not invariably lead to loneliness. Indeed, for religious leaders such as the Buddha, disengagement from the social world was a prerequisite for inner peace and spiritual transcendence. Some of the most esteemed creative luminaries have harnessed the benefits of alone time for their artistic craft; Pablo Picasso once said that "no serious work is possible without great solitude" (Cain 2012). Everyday people, too, may reap the benefits of solitude; according to nation-wide surveys, $85 \%$ of adults view spending time alone as very important to them (Madden and Rainie 2015), and a majority of individuals wish they had more time to themselves (Larson et al. 1982).

These and other accounts point to the possibility of two different ways of experiencing aloneness: loneliness versus solitude. These experiences may be differentiated based on their valence (Long and Averill 2003). On one hand, loneliness describes a negative emotional state characterized by a perceived gap between our desired and actual social contact (Masi et al. 2011). On the other hand, solitude describes a neutral or positive emotional state that involves a "disengagement from the immediate demands of other people" (Long and Averill 2003, p. 23).

Although loneliness negatively impacts our health, solitude is associated with a host of psychological benefits. Solitude promotes positive psychological adjustment, stress management, and life satisfaction (Larson 1997; Larson and Lee 1996; Leary et al. 2003). In addition, solitude can powerfully boost affective regulation by reducing the magnitude of arousal, whether such arousal is associated 
with positive or negative valence (Nguyen et al. 2018). Preliminary research even suggests that periods of solitude may enhance the quality of our relationships (Ben-Ari 2012) and stimulate our creativity (Nguyen et al. 2018).

\section{The Cognitive Approach and Time Spent Alone}

What determines whether time alone is experienced positively or negatively? Cognitive theories suggest that our appraisals of time alone may determine whether we experience solitude or loneliness. In this view, if we appraise time alone as positive, we may experience solitude and its associated benefits. By contrast, if we appraise our time alone as negative, then we may fall victim to the pains of loneliness.

What factors may lead us to negatively appraise our time alone? Previous research suggests that self-stigma, distorted social cognition, and social media use may contribute to negative appraisals of time alone. First, self-stigma (the internalization of negative social appraisal of a personal characteristic or behavior) around loneliness may place us at greater risk for experiencing loneliness ourselves (Rotenberg and MacKie 1999). Research suggests that individuals tend to view loneliness as a sign of personal weakness or inadequacy. In two separate studies, participants asked to judge hypothetical peers were significantly less accepting of peers described as "lonely" than those described as "nonlonely" (Lau and Gruen 1992; Rotenberg and MacKie 1999). Lonely peers were consistently rated as less competent, less attractive, less psychologically adjusted, and less desirable as friends (Lau and Gruen 1992; Rotenberg and MacKie 1999). This stigma may undermine our own capacity for fruitful time alone by causing us to appraise it negatively.

Second, distorted social cognition may also increase our risk of loneliness. Research suggests that we tend to underestimate how lonely others feel and overestimate how happy they are (Jordan et al. 2011). Indeed, individuals who believe that other people have richer social lives than they do (e.g., attend more parties, have more friends, and encounter fewer social obstacles) report greater feelings of loneliness (Deri et al. 2017; Jordan et al. 2011). Thus, viewing our social lives as inferior to those of others to may lead us to feel dissatisfied with our own social lives and, consequently, more likely to appraise time alone as loneliness.

Finally, self-stigma and distortion of social cognition may be amplified by particular forms of social media use (Verduyn et al. 2017). As social media platforms often present a positively curated version of reality (e.g., showcasing "highlight reels" of socially rewarding moments from others' lives), passive use of social media (e.g., scrolling on one's feed) use may perpetuate maladaptive social cognition and fuel loneliness (Aalbers et al. 2019; Liu et al. 2017; Verduyn et al. 2015). Specifically, engagement in upward social comparison (i.e., comparing ourselves to those we believe have more social capital) may render us dissatisfied with our social lives and more vulnerable to loneliness (Kross et al. 2013; Wang et al. 2017; Yang 2016). Taken together, this research suggests that excessive or passive social media use may fuel negative social cognition and lead us to negatively appraise our time alone. However, the effect of social media use on our appraisals of time spent alone is as of yet unclear, and experimental research is needed.

Other types of appraisal less related to interpersonal factors may also contribute to loneliness. Even a seemingly easy task - sitting alone for 15 min — can be deeply uncomfortable for many. In one study, participants were offered the choice of sitting alone in a lab room for 15 min or experiencing a mild electric shock (Wilson et al. 2014). Notably, most participants chose the electric shock. It is uncertain whether this choice indicated a fear of loneliness specifically, or an aversion to boredom and idleness (Wilson et al. 2014). Indeed, individuals who are more prone to boredom are also more likely to feel lonely (Skues et al. 2016). More research is needed to clarify the role of non-social appraisals of isolation, as well as the discriminability of loneliness from other phenomena such as a idleness.

\section{Cognitive Reappraisal and Time Spent Alone}

Fortunately, cognitive theories of emotion suggest that with practice, we can alter our negative appraisals of life events (Cacioppo and Patrick 2008). An emotional regulation strategy known as cognitive reappraisal, which involves reinterpreting the meaning of a situation, can help us generate more adaptive emotional responses to distressing situations (Troy et al. 2010). The benefits of cognitive reappraisal have been documented extensively in the context of arousal. Previous research has found that reappraising physiological arousal (i.e., increased heart rate, blood pressure, and respiration) helps improve academic performance. In one study, participants who read a short passage that described arousal as beneficial to performance scored significantly higher than a control group on the Graduate Record Examination (GRE) (Jamieson et al. 2010). Individuals who more positively reappraised their arousal also experienced lower stress during the exam than those who negatively appraised their arousal (Jamieson et al. 2010).

These findings raise the question of whether cognitive reappraisal might be useful in other contexts, such as changing our relationship with our time alone. As cognitive reappraisal has found to be most useful when a situation cannot be changed or acted upon, it may be an especially viable tool for addressing isolation that cannot be remedied by immediate resumption of social contact (Gross and John 2003). There is reason to believe that addressing maladaptive beliefs through cognitive reappraisal would enable 
individuals to experience their time alone more positively, and ultimately, reap the benefits of solitude and avoid the pains of loneliness. Although the link between maladaptive social cognition and loneliness has been established, no study to date has determined whether our subjective experience of time alone can be changed.

\section{The Current Study}

The current study investigates whether a brief cognitive reappraisal manipulation enables individuals to experience a subsequent period of time alone more positively (i.e., reappraise their time alone as solitude rather than loneliness). Further, we sought to determine whether different types of reappraisal manipulations might have differential effects on the experience of time alone. To this end, we conducted a randomized controlled experiment with a control condition, and two different experimental conditions. The first experimental condition sought to induce a reappraisal of time alone by framing it as a positive state of solitude. In this condition, participants read a short passage about the benefits of solitude (Solitude Benefits condition). The second experimental condition sought to induce a reappraisal of being alone by correcting the perception of loneliness as uncommon or pejorative. In this condition, participants read a short passage about the high prevalence of loneliness in the population (Loneliness De-Biasing condition). We also took into account the possible role of other variables theoretically relevant to appraisals of time alone: trait loneliness, compulsive social media use, and trait reappraisal. We now address each specific aim in turn.

\section{Aim 1: Can a Reappraisal Enable a more Positive Experience of Time Alone?}

In Jamieson et al.'s (2010) study, participants who reframed physiological arousal as positive experienced significantly less stress during a subsequent exam. It is possible, then, that reframing time alone as positive will increase positive mood after a brief period of time alone. Therefore, we expected participants in the Solitude Benefits condition to report an increase in positive mood after sitting alone relative to the control condition (Hypothesis I). Given the potential for the Loneliness De-Biasing condition to prime participants to feel lonely (due to its references to loneliness in the population as a whole), we did not think it would induce an increase in positive mood. However, we did expect participants in the Loneliness De-Biasing condition to experience a decrease in negative mood, as it would attenuate self-stigma thought to be generative of loneliness. Thus, we expected individuals in both reappraisal conditions to experience a reduction in negative mood relative to participants in the Control condition (Hypothesis II).

\section{Aim 2: Is the Effect of Reappraisal Moderated by Trait Loneliness, Compulsive Social Media Use, or Trait Reappraisal?}

As trait loneliness, compulsive social media use, and trait reappraisal are theoretically relevant to the cognitive factors involved in time spent alone, there is reason to believe they might influence individuals' success in reappraising time alone. We hypothesized that all three of these factors would moderate the effects of the reappraisal manipulations in the following ways.

First, we predicted that for individuals higher in trait loneliness, the previously hypothesized effect of the Solitude Benefits condition on positive mood would be attenuated, as would the effect of both reappraisal manipulations on negative mood (Hypothesis III). We predicted this moderating effect because lonelier individuals may have a more difficult time reappraising time alone, as they are more accustomed to appraising it negatively. Second, we predicted that greater compulsive social media use would similarly attenuate the hypothesized effects of the reappraisal manipulations (Hypothesis IV). We predicted this effect because heavy social media users may more often encounter material generative of maladaptive social cognition (i.e., distressing upward social comparisons), or may more often turn to social media to distract themselves from the distress of time alone. Thus, they may have a harder time enjoying a 10-min period of time alone. Third, we predicted that greater trait reappraisal would augment the hypothesized effects of our reappraisal manipulations (Hypothesis V). We predicted this effect because individuals who more routinely utilize cognitive reappraisal in their everyday lives may find it easier to apply such skills to time alone.

\section{Exploratory Aim 3: How are Loneliness, Age, Cognitive Styles, and Different Types of Social Media Use Related to One Another?}

As an exploratory aim, we were interested in examining how our outcome and moderator variables relate to one another in our sample. Given the literature implicating other aspects of social media use in the risk for loneliness in younger age groups, we also included other aspects of social media use potentially relevant to loneliness (i.e., engagement in upward social comparison on social media, active versus passive social media use) as well as participant age. As this research aim was exploratory, we did not develop any a priori hypotheses. 


\section{Method}

\section{Participants}

All recruitment materials, stimuli, $\mathrm{R}$ code, and the de-identified dataset can be found at the Open Science Framework (OSF) web page for this study at osf.io/wsv3y/. All procedures received institutional approval from the Harvard University Committee on the Use of Human Subjects. College students and community members were recruited via the Harvard University Psychology Department's research recruitment website during the fall semester of 2019. The website advertised the study under the title "Emotions, Social Media, and You" and did not reference loneliness in the study description. Study advertisement materials can be found in supplementary materials $\mathrm{S} 1$. To be eligible for the 30-min laboratory study, participants had to be fluent in English and 18 years of age or older. Participants received either \$5 USD in cash or 0.5 psychology study pool credits for their time.

A total of 243 participants completed the study. Prior to examining data, 23 participants were excluded from analyses due to non-compliance with study procedures ${ }^{1}(n=15)$, falling asleep during the waiting period $(n=4)$, or missing data/invalid responding $(n=4)$. The final sample used for analyses included 220 participants who had been randomly assigned into either the Solitude Benefits condition $(n=74)$, Loneliness De-Biasing condition $(n=72)$, or Control condition $(n=74)$.

\section{Measures}

\section{Outcome Measures}

Positive and Negative Affect Schedule The Positive and Negative Affect Schedule (PANAS) (Watson et al. 1988) is a widely validated tool for measuring state mood. The PANAS comprises a positive affect subscale (PANAS-P) and a negative affect subscale (PANAS-N). Each subscale contains ten affectively valenced descriptors (e.g. inspired, jittery, enthusiastic, distressed). Participants rated the degree to which they felt each emotional state in the present moment on a 5 -point Likert scale $(1=$ not at all, $5=$ very much) at two different time points: before (Time 1) and after (Time 2) the manipulation. In our sample, both subscales displayed very good internal consistency [PANAS-P:

\footnotetext{
${ }^{1}$ Non-compliance with study procedures includes calling the experimenter during the waiting period $(n=8)$, browsing the internet on the laboratory computer $(n=6)$, and using a smartwatch during the study $(n=1)$
}

$\alpha=0.89$ (Time 1), $\alpha=0.91$ (Time 2); PANAS-N: $\alpha=0.87$ (Time 1), $\alpha=0.89$ (Time 2)].

\section{Moderators}

UCLA Loneliness Scale-Revised, 3rd Edition The UCLA Loneliness Scale, 3rd edition (Russell 1996) is a widely used measure of perceived social isolation. Participants indicated the extent to which they have experienced various aspects of loneliness (e.g. I feel isolated from others) on a 4-point Likert scale $(1=$ never; $4=$ often $)$. The scale has demonstrated convergent validity in its robust associations with other measures of loneliness (Russell 1996). We used a shortened 8-item version of the scale which has similar psychometric properties to the original 20-item version (Russell 1996). The scale showed adequate internal consistency in our sample $(\alpha=0.74)$.

Compulsive Social Media Use Scale The Compulsive Internet Use Scale (Meerkerk et al. 2009) assesses the severity of compulsive internet use and the degree to which internet use interferes with everyday functioning. We modified this scale to ask about behaviors specific to "social media" use rather than internet use more generally (e.g. How often have you unsuccessfully tried to spend less time on social media?). We also shortened the scale from fourteen to eight items, removing redundant items for brevity. Items are scored on a 6-point Likert scale $(1=$ never, $6=$ very often $)$, with larger aggregate scores indicating greater compulsive social media use tendencies. The Compulsive Social Media Use Scale displayed acceptable internal consistency in our sample $(\alpha=0.79)$. See supplementary materials $\mathrm{S} 2$ for our modified version of the scale.

Emotion Regulation Questionnaire-Reappraisal Subscale The Emotion Regulation Questionnaire (ERQ) (Gross and John 2003) measures how often individuals use two emotion regulation techniques (i.e., cognitive reappraisal and expressive suppression) in their everyday lives. Because our study examines cognitive reappraisal, we administered only the reappraisal subscale. Participants rated their responses to six items (e.g. I control my emotions by changing the way I think about the situation I'm in) on a 7-point Likert scale $(1=$ strongly disagree, $7=$ strongly agree $)$. Higher total scores indicate greater trait cognitive reappraisal. The ERQReappraisal subscale showed adequate internal consistency in our sample $(\alpha=.73)$.

\section{Other Measures}

Social Media Usage Questionnaire We developed this brief questionnaire for the purpose of this experiment (see supplementary materials S2). Participants were asked a series 
of questions about their daily social media use. First, participants reported the average number of hours they spend on social media each day. Then, participants were asked to estimate what portion of the stated time is used for posting, scrolling, commenting, and messaging others directly. Responses were divided into two categories: passive social media use (i.e., total time spent scrolling) and active social media use (i.e., sum of time spent posting, commenting, and direct messaging), enabling a fine-grained estimate of participants' engagement in passive vs. active social media use.

Social Comparison Scale The Social Comparison Scale (Allan and Gilbert 1995) assesses how individuals perceive themselves in relation to others. We modified the instructions and asked participants to indicate their self-perceptions when using social media (as opposed to general selfperceptions). The scale consists of eleven pairs of opposing constructs (e.g. inferior vs. superior, insider vs. outsider). Participants were asked to rate themselves on a continuum between pairs of constructs on a scale from 1 (highest rating on first extreme of construct) to 10 (highest rating on second extreme of construct). Items were reverse-scored such that higher scores indicated greater upward social comparison. The Social Comparison Scale showed good internal consistency in our sample $(\alpha=.89)$.

Waiting Period Activities Questionnaire We asked a series of questions about participants' thoughts and feelings during the waiting period, most of which were designed to answer questions outside the scope of this article. However, one question (i.e., My beliefs about time alone were challenged) rated on a 7-point Likert scale $(1=$ strongly disagree, $7=$ strongly agree) served as a manipulation check for the reappraisal conditions.

Demographics Questionnaire This questionnaire asked participants to report their age, gender, sexual orientation, ethnicity, and highest level of education.

\section{Materials}

Participants were randomly assigned to read one of three informative passages. For consistency, passages were standardized in length (between 195 and 205 words) and content (i.e., contained both statistical data and research-backed evidence). All passages can be found in supplementary materials S3.

The Solitude Benefits passage framed time alone as a positive experience with a variety of potential benefits; i.e., boosts in emotion regulation, enhanced creativity, and improved mental well-being. The Loneliness DeBiasing passage framed loneliness as a natural part of the human experience and challenged the erroneous belief that loneliness is rare or disgraceful. The Control passage described the Harvard University research recruitment website and was unrelated to loneliness (the words "loneliness" and "solitude" were not mentioned).

\section{Procedure}

Upon arrival at the laboratory site, participants were greeted by the experimenter. ${ }^{2}$ Participants were asked to leave all personal belongings and electronic devices (e.g. cellphones, smartwatches, laptops, Fitbits) in a separate room. Once in the testing room, participants received, read, and signed an institutionally approved consent form with detailed information about the study and the specific activities involved.

After providing informed consent, participants completed a battery of baseline measures on an online Qualtrics survey administered from a laboratory computer. The measures administered were the PANAS, Compulsive Social Media Use Scale, Social Media Usage Questionnaire, Social Comparison Scale, ERQ-Reappraisal Subscale, and UCLA Loneliness Scale-Shortened Form.

Next, participants were randomly assigned to read one of three written passages (Solitude Benefits, Loneliness De-Biasing, or Control). Then, the survey informed participants of a 10-min sitting alone task, described as a "waiting period" in order to mask the true purpose of the study. The Qualtrics survey automatically began a 10-min timer and advanced to the next page after the $10 \mathrm{~min}$ had elapsed. Following the waiting period, participants were prompted to fill out the PANAS for a second time, as well as the Waiting Period Activities questionnaire and the demographics questionnaire.

Finally, the experimenter fully debriefed participants about the purpose of the experiment and provided participants with a debriefing form to take home. Participants received compensation and were released.

\section{Planned Analyses}

Our final sample size $(N=220)$ provided sufficient power $(1-\beta$ error probability $=.998)$ to detect a small effect size $\left(\eta_{\mathrm{p}}{ }^{2}=.01\right)$ in our analyses of variance. First, we planned to examine sample characteristics and conduct conditionwise group comparisons on relevant variables to determine the success of the randomization to condition. As a preliminary analysis, we planned to conduct a series of paired sample $t$-tests to determine whether positive and negative mood changed significantly from Time 1 and Time 2 (preand post-manipulation) in each condition. In line with our

\footnotetext{
${ }^{2}$ Four experimenters (the first author and three undergraduate research assistants) ran participants for this study.
} 
exploratory Aim 3, we conducted a series of bivariate correlations (Pearson's $r$ ) to assess the zero-order associations between our proposed moderator variables, participant age, and other indices of social media use (tendency toward upward social comparison on social media, passive and active social media use).

To test Hypotheses I and II, we first subtracted each participant's positive mood (PANAS-P score) and negative mood (PANAS-N score) at Time 1 from their positive and negative mood at Time 2, yielding change scores for each participant for both positive and negative mood. We then conducted a series of ANOVAs to determine whether changes in positive and negative mood differed significantly across conditions. In the case of significant differences in either set of analyses, we planned to conduct Tukey's post-hoc tests to determine the direction and magnitude of the differences. As a manipulation check, we also ran an ANOVA to examine whether condition significantly impacted the degree to which participants' beliefs about their time alone were challenged.

To test Hypotheses III, IV, and V, we conducted six multiple regressions to assess whether trait loneliness, compulsive social media use, and trait reappraisal moderate the relationships between condition and changes in either positive or negative mood. For each regression, we included condition as a dummy-coded independent variable (Control $=0$, Loneliness De-Biasing $=1$, Solitude Benefits $=2$ ), the proposed moderator as a continuous independent variable, and the cross-product of condition and the proposed moderator as an independent variable. If any of the cross-product terms were statistically significant, we planned to conduct a simple slopes analysis to interpret the effect of condition on changes in mood at varying levels of the moderator. See supplementary materials $\mathrm{S} 4$ for all $\mathrm{R}$ code used in our analyses and supplementary materials S5 for the de-identified dataset.

Finally, we used mean imputation for single instances of missing responses.

\section{Results}

\section{Sample Characteristics}

The mean age of participants was 25.93 years old $(S D=11.6$ years, range: $18-73)$. Gender in our sample was $65.0 \%$ female $(n=143), 34.1 \%$ male $(n=75)$, and $0.9 \%$ gender non-binary $(n=2)$. Participants identified their ethnicity as either White $(45.9 \% ; n=101)$, Latino $(9.5 \% ; n=21)$, Asian $(29.1 \% ; n=64)$, Black $(8.2 \% ; n=18)$, or other $(7.3 \%$; $n=16$ ). All participants had at least a high school diploma, and a considerable proportion of participants had either completed some college $(n=96,43.6 \%)$ or attained a bachelor's degree $(n=49,22.3 \%)$.
Table 1 displays sample characteristics and outcome variables by condition. Gender, ethnicity, sexual orientation, and level of education did not differ significantly across conditions. However, age did differ significantly by condition $(F(2,217)=3.27, p=.04)$. Participants in the Solitude Benefits condition were slightly older than participants in the Control condition according to a Tukey post-hoc test $\left(M_{\text {Difference }}=4.51, p<.05\right)$. Therefore, we controlled for age by including it as a covariate in all of our analyses of variance and multiple regressions.

\section{Manipulation Check}

Condition significantly affected the degree to which the manipulation challenged participants' beliefs about their time alone $(F(2,217)=3.46, p=.03)$. A post-hoc analysis with the Tukey adjustment showed that the Solitude Benefits passage more strongly challenged participants' beliefs about time alone than did the Control passage $\left(M_{\text {Difference }}=0.43\right.$, $p=.03)$. However, there were no significant differences between the Loneliness De-Biasing condition and either the Control condition $\left(M_{\text {Difference }}=0.15 ; p=.62\right)$ or the Solitude Benefits condition $\left(M_{\text {Difference }}=-0.28 ; p=.23\right)$.

\section{Preliminary Analyses}

Across conditions, participants experienced significant reductions in both positive mood (Solitude Benefits, $t(74)=3.62, p<.001$; Loneliness De-Biasing, $t(72)=6.68$, $p<.001$; Control, $t(73)=6.98, p<.001)$ and negative mood (Solitude Benefits, $t(74)=4.19, p<.001$; Loneliness DeBiasing, $t(72)=2.35, p=.02$; Control, $t(74)=5.46, p<.001$ ) after sitting alone for $10 \mathrm{~min}$.

\section{Bivariate Correlations}

Table 2 displays a matrix of bivariate correlations between proposed moderator variables, other aspects of social media use, and participant age. Participant age was negatively correlated with compulsive social media use and upward social comparison on social media. However, age was not significantly correlated with either loneliness or daily time spent on social media. Trait loneliness showed a moderate positive correlation with compulsive social media use and upward social comparison on social media. Trait loneliness was negatively associated with trait reappraisal. Trait loneliness was not significantly correlated with either total, active, or passive social media use. Finally, trait reappraisal was negatively correlated with compulsive social media use and upward social comparison on social media. 
Table 1 Demographic and baseline characteristics by condition

\begin{tabular}{|c|c|c|c|}
\hline Variable & Control $(n=74)$ & $\begin{array}{l}\text { Loneliness de-biasing } \\
(n=72)\end{array}$ & $\begin{array}{l}\text { Solitude benefits } \\
(n=74)\end{array}$ \\
\hline Age $(M, S D)$ & $24.26(9.06)$ & $24.97(9.49)$ & $28.77(15.01)$ \\
\hline \multicolumn{4}{|l|}{ Gender $(n, \%)$} \\
\hline Female & $50(67.6 \%)$ & $53(73.6 \%)$ & $40(54.1 \%)$ \\
\hline Male & $23(31.1 \%)$ & $18(25.0 \%)$ & $34(45.9 \%)$ \\
\hline Non-binary & $1(1.4 \%)$ & $1(1.4 \%)$ & $0(0.0 \%)$ \\
\hline \multicolumn{4}{|l|}{ Ethnicity $(n, \%)$} \\
\hline White & $26(35.1 \%)$ & $36(50.0 \%)$ & $39(52.7 \%)$ \\
\hline Hispanic & $9(12.2 \%)$ & $8(11.1 \%)$ & $4(5.4 \%)$ \\
\hline Asian & $22(29.7 \%)$ & $25(34.7 \%)$ & $17(23.0 \%)$ \\
\hline Black & $9(12.2 \%)$ & $1(1.4 \%)$ & $8(10.8 \%)$ \\
\hline Other & $8(10.8 \%)$ & $2(2.8 \%)$ & $6(8.1 \%)$ \\
\hline \multicolumn{4}{|l|}{ Education level $(n, \%)$} \\
\hline High school & $22(29.7 \%)$ & $13(18.1 \%)$ & $14(18.9 \%)$ \\
\hline Some college & $27(36.5 \%)$ & $31(43.1 \%)$ & $37(50.0 \%)$ \\
\hline Associate's & $1(1.4 \%)$ & $2(2.8 \%)$ & $2(2.7 \%)$ \\
\hline Bachelor's & $17(23.0 \%)$ & $18(25.0 \%)$ & $15(20.3 \%)$ \\
\hline Master's & $6(8.1 \%)$ & $8(11.1 \%)$ & $3(4.1 \%)$ \\
\hline Professional & $1(1.4 \%)$ & $0(0.0 \%)$ & $3(4.1 \%)$ \\
\hline \multicolumn{4}{|l|}{ Baseline $(M, S D)$} \\
\hline Daily SMU & $2.34(1.63)$ & $2.14(1.34)$ & $2.29(1.84)$ \\
\hline Compulsive SMU & $21.88(5.42)$ & $22.35(6.13)$ & $21.69(5.46)$ \\
\hline Social comparison & $63.07(15.30)$ & $63.69(15.87)$ & $62.91(15.51)$ \\
\hline Trait reappraisal & $21.99(3.65)$ & $21.82(3.40)$ & $20.92(4.28)$ \\
\hline Trait loneliness & $18.23(4.88)$ & $17.43(4.33)$ & $18.59(5.32)$ \\
\hline \multicolumn{4}{|l|}{ State $\operatorname{mood}(M, S D)$} \\
\hline \multicolumn{4}{|l|}{ Time 1} \\
\hline PANAS-P ${ }^{\mathrm{a}}$ & $27.04(7.77)$ & $24.82(6.90)$ & $26.54(7.31)$ \\
\hline PANAS- $\mathrm{N}^{\mathrm{b}}$ & $14.81(6.08)$ & $14.06(4.67)$ & $14.92(5.67)$ \\
\hline \multicolumn{4}{|l|}{ Time 2} \\
\hline PANAS-P & $23.15(9.23)$ & $21.65(6.44)$ & $24.82(8.09)$ \\
\hline PANAS-N & $13.26(5.11)$ & $13.38(5.10)$ & $13.65(5.73)$ \\
\hline \multicolumn{4}{|l|}{ Mood change } \\
\hline PANAS-P ${ }_{\mathrm{T} 2-\mathrm{T} 1}$ & $-3.89(5.21)$ & $-3.17(4.43)$ & $-1.72(4.87)$ \\
\hline PANAS- $\mathrm{N}_{\mathrm{T} 2-\mathrm{T} 1}$ & $-1.55(2.97)$ & $-0.68(3.62)$ & $-1.27(3.29)$ \\
\hline
\end{tabular}

There was a significant difference in age across conditions; $F(2,217)=3.27, p<.05$

${ }^{\mathrm{a}}$ PANAS-P PANAS Positive Affect Subscale, ${ }^{\mathrm{b}}$ PANAS- $N$ PANAS Negative Affect Subscale

\section{Main Analyses}

Prior to analysis, we assessed homogeneity of variance in changes in positive and negative mood across conditions. According to Levene's test, neither changes in positive mood $(F(2,217)=.15, p=.86)$ nor changes in negative $\operatorname{mood}(F(2$, $217)=.05, p=.95)$ violated the homogeneity of variance assumption.

\section{Hypothesis I: Effect of Manipulation on Changes in Positive Mood $^{3}$}

We found a small but significant effect of condition on changes in positive $\operatorname{mood}\left(F(2,216)=3.87, p=.02, \eta_{\mathrm{p}}{ }^{2}=.03\right)$. A

\footnotetext{
3 At the suggestion of an anonymous reviewer of a previous version of this manuscript, we assessed the effect of condition on positive and negative mood at Time 2 (post-manipulation) in two separate ANOVAs. The results of these analyses can be found in Supplementary Materials S6 online at https://osf.io/wsv3y/.
} 
Table 2 Bivariate correlations between outcomes of interest $(N=220)$

\begin{tabular}{llclllll}
\hline & Age & UCLA & CSMU & USC & ERQ & SMU total & SMU passive \\
\hline UCLA & -.06 & - & & & & & \\
CSMU & $-.32^{* * *}$ & $.31^{* * *}$ & - & & & \\
USC & $-.18^{* *}$ & $.38^{* * *}$ & .06 & - & & \\
ERQ & .03 & $-.18^{* *}$ & $-.22^{* * *}$ & $-.17^{*}$ & - & & \\
SMU-total & -.02 & .06 & $.33^{* * *}$ & $-.16^{*}$ & $-.15^{*}$ & - & \\
SMU-passive & -.10 & .05 & $.19^{* *}$ & -.05 & $-.17^{*}$ & $.59^{* * *}$ & - \\
SMU-active & .02 & .02 & $.24 * * *$ & $-.18^{* *}$ & -.01 & $.60^{* * *}$ & -.01
\end{tabular}

UCLA trait loneliness, CSMU compulsive social media use, USC upward social comparison (social comparison scale-reverse scored), ERQ trait reappraisal, $S M U$-Total total daily social media use, SMU-Passive daily passive social media use, $S M U$-Active daily active social media use

$* * * p<.001, * * p<.01, * p<.05$

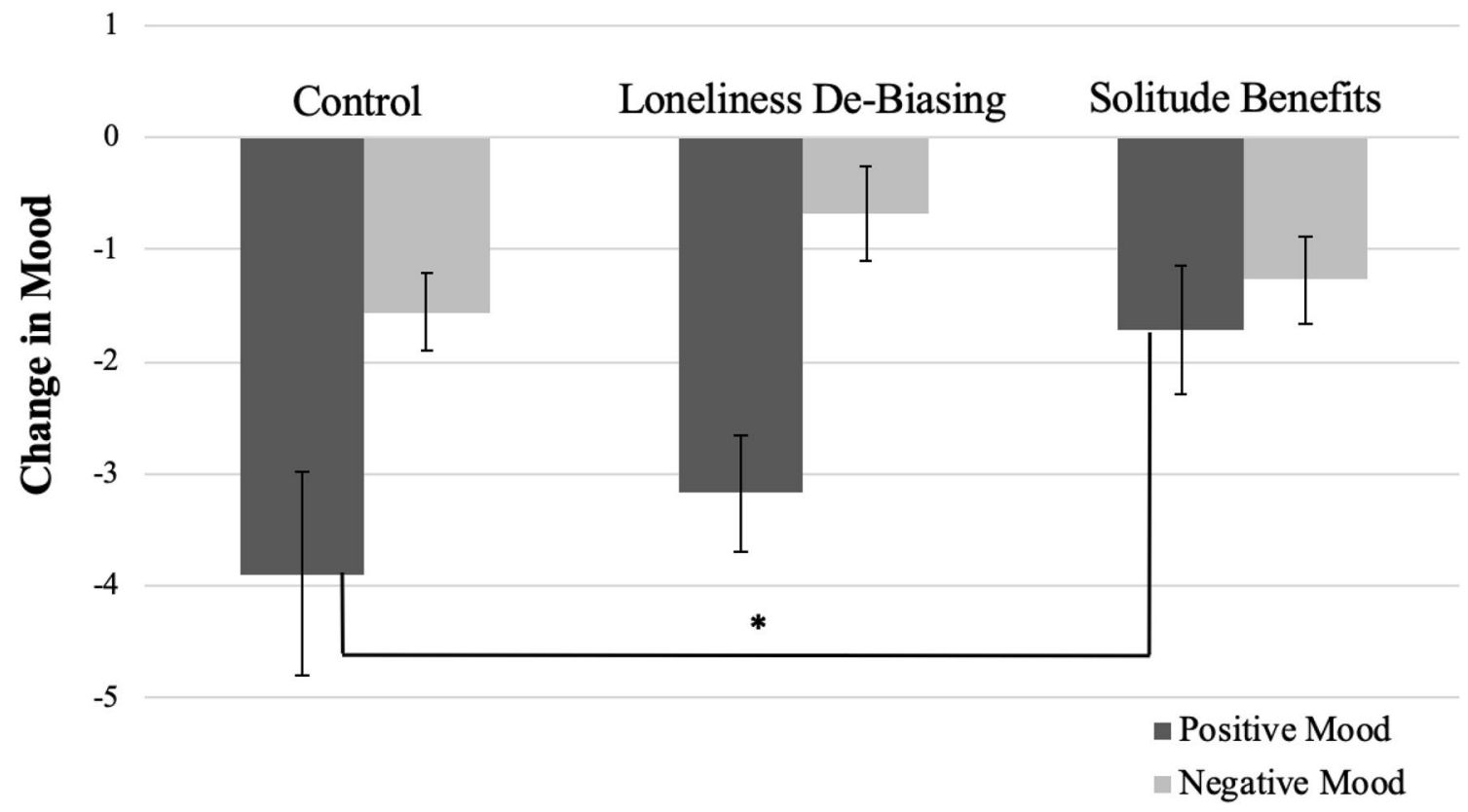

Fig. 1 Changes in positive and negative mood from time 1 to time 2 by condition

post-hoc analysis with the Tukey adjustment revealed that positive mood decreased significantly less in the Solitude Benefits condition than in the Control condition $\left(M_{\text {Difference }}=2.17\right.$, $p=.02)$. Changes in positive mood did not significantly differ between the Loneliness De-Biasing condition and either the Solitude Benefits condition $\left(M_{\text {Difference }}=1.45, p=.17\right)$ or the Control condition $\left(M_{\text {Difference }}=-0.72, p=.64\right)$. See Fig. 1 for a graph of changes in positive mood by condition.

\section{Hypothesis II: Effect of Manipulation on Changes in Negative Mood}

We did not find a significant effect of condition on changes in negative $\operatorname{mood}(F(2,216)=1.32, p=.27)$. See Fig. 1 for a graph of changes in negative mood by condition.

\section{Moderation Analyses}

\section{Hypothesis III: Trait Loneliness as a Moderator of Mood Change}

The regression to detect an interaction between trait loneliness and condition in predicting change in positive mood did not yield significant cross-products between trait loneliness and either the Loneliness De-Biasing condition $(t(213)=1.75, p=.08)$ or the Solitude Benefits condition $(t(213)=1.81, p=.07) ; F(6,213)=2.32, p=.03, R^{2}=.06$. An analogous interaction detection with changes in negative mood as the dependent variable also did not yield significant cross-products between trait loneliness and either the Loneliness De-Biasing condition $(t(213)=-.17, p=.86)$ or 


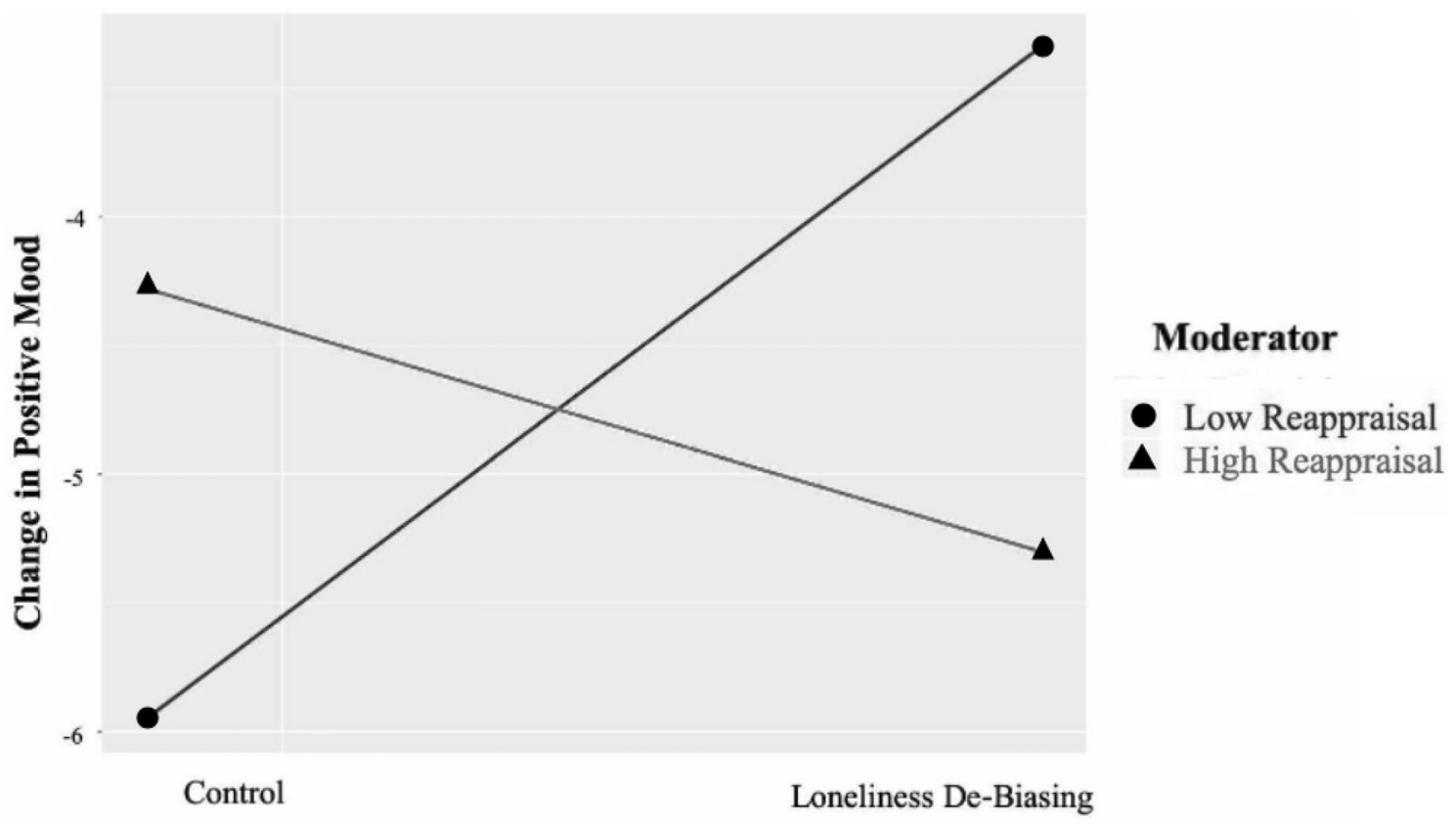

Condition

Fig. 2 Conditional effects of the Loneliness De-Biasing condition on positive mood change at high (+ $1 S D)$ and low (- $1 S D)$ levels of trait reappraisal

the Solitude Benefits condition $(t(213)=.27, p=.79) ; F(6$, 213) $=1.33, p=.24, R^{2}=.04$.

\section{Hypothesis IV: Compulsive Social Media Use as a Moderator of Mood Change}

The regression to detect an interaction between compulsive social media use on and condition on change in positive mood did not yield significant cross-products between compulsive social media use and either the Loneliness De-Biasing condition $(t(213)=.27, p=.79)$ or the Solitude Benefits condition $(t(213)=1.20, p=.23) ; F(6,213)=2.24, p=.04$, $R^{2}=.06$. An analogous interaction detection with changes in negative mood as the dependent variable also did not yield significant cross-products between compulsive social media use and either the Loneliness De-Biasing condition $(t(213)=1.30, p=.20)$ or the Solitude Benefits condition $(t(213)=-.12, p=.91) ; F(6,213)=1.38, p=.22, R^{2}=.04$.

\section{Hypothesis V: Trait Reappraisal as a Moderator of Mood Change}

The regression to detect an interaction between trait reappraisal and condition on change in positive mood yielded significant cross-products between trait reappraisal and the Loneliness De-Biasing condition $(t(213)=-2.2, p=.03)$, but not between trait reappraisal and the Solitude Benefits condition $(t(213)=0.01, p=.99) ; F(6,213)=2.75, p=.01$, $R^{2}=.04$.

To examine this interaction further, we conducted a simple slopes analysis to determine the effect of condition on change in positive mood at one $S D$ above and below the mean level of trait reappraisal (see Fig. 2). For individuals high in trait reappraisal, there was no significant effect of the Loneliness De-Biasing manipulation on change in positive $\operatorname{mood}(t(141)=-0.99, p=.32, b=1.13)$. However, for those low in trait reappraisal, the Loneliness DeBiasing manipulation did effect a significantly smaller decrement in positive mood relative to the Control condition $(t(141)=2.19, p=.03, b=1.12)$.

An analogous interaction detection including changes in negative mood as a dependent variable did not yield significant cross-products between trait reappraisal and either the Loneliness De-Biasing condition $(t(213)=-1.16, p=.25)$ or the Solitude Benefits condition $(t(213)=.11, p=.91)$; $F(6,213)=.98, p=.44, R^{2}=.03$.

\section{Discussion}

This study is the first to examine whether the benefits of cognitive reappraisal may apply to time spent alone. We now discuss each set of analyses in turn and examine how our findings reflect on our hypotheses. 


\section{Preliminary Analyses}

Across conditions, participants' positive and negative mood significantly decreased after sitting alone for $10 \mathrm{~min}$. This is consistent with previous research showing that spending time alone can reduce affective intensity (Nguyen et al. 2018). Although time alone may not necessarily increase positive mood, it may decrease the magnitude of arousal for both positive and negative affect, thereby promoting emotional regulation (Nguyen et al. 2018).

\section{Bivariate Correlations}

Our bivariate correlations illuminate potentially important distinctions in how loneliness, cognitive styles, and social media relate to one another. Our findings suggest that younger people use social media more compulsively and engage more in upward social comparison on these platforms than older people do. As most young adults have been exposed to digital technology since childhood, they may have developed a habitual use of social media earlier in life (Twenge et al. 2019). In addition, younger individuals may have a greater investment in online social standing and thus display more compulsive behaviors when using social media than their older counterparts. Our findings also suggest that social media use may have its most pernicious effects on loneliness by virtue of the opportunities for upward social comparisons that it affords. These results align with previous research indicating that particular behaviors on social media, rather than the total amount of time spent on social media, places us at greater risk for loneliness (Verduyn et al. 2017; Yang 2016).

Surprisingly, loneliness was not significantly correlated with passive social media use, diverging from previous findings that passive social media use predicts greater loneliness (Aalbers et al. 2019; Verduyn et al. 2015). This discrepancy may arise from the way in which we measured passive social media use (via self-report). Indeed, self-reports may be subject to recall biases, and individuals may engage in passive social media use habitually or without real awareness (Aalbers et al. 2019). By contrast, Aalbers et al. (2019) collected data across 2 weeks through an experience sampling protocol and thus obtained a more ecologically valid measure of passive social media use.

Finally, we found that trait reappraisal was negatively associated with loneliness, compulsive social media use, and upward social comparison. These findings align with research showing that greater use of cognitive reappraisal can serve as a protective factor against negative emotion in the face of stress (Troy et al. 2010). Our exploratory analyses were cross-sectional, and cannot confirm causality. However, they do provide specific distinctions regarding points of intersection among loneliness, cognitive styles, and social media use. These distinctions may prove to be fruitful avenues for further research.

\section{Hypothesis I: Effect of Manipulation on Changes in Positive Mood}

Contrary to our predictions, participants who read about the benefits of solitude did not experience an increase in positive mood after sitting alone for $10 \mathrm{~min}$. However, these participants did experience a smaller reduction in positive mood after sitting alone than did participants in the Control condition. In other words, reframing time alone as solitude boosted resilience to decrements in positive mood otherwise associated with time alone, providing partial support for Hypothesis I.

Importantly, our manipulation showed an effect on the cognitive process proposed to be operative in our experiment: reappraisal. Individuals who read about the benefits of solitude reported that their beliefs about time alone were challenged more than did those who read a control passage. The fact that reading two brief paragraphs about the potential benefits of solitude significantly challenged beliefs about time alone supports our initial assumption that people, on average, do not view time alone as positive. By contrast, reading about the high prevalence of loneliness neither significantly challenged individuals' beliefs about their time alone nor induced a reappraisal of time alone.

It is surprising that such a brief manipulation (i.e., a short reading task) was sufficient to change how individuals experience their time alone. The size of our effect, albeit small, echoes previous findings demonstrating the success of brief cognitive reappraisal manipulations in regard to physiological arousal (Jamieson et al. 2010). Taken together, these findings support the idea that our appraisals of life situations are flexible, rather than fixed, and promote reappraisal as a powerful mechanism for emotional regulation (Troy et al. 2010).

\section{Hypothesis II: Effect of Manipulation on Changes in Negative Mood}

Neither manipulation showed an effect on changes in negative affect relative to the control condition. Thus, our Hypothesis II was not supported. There are several possible reasons for why the Loneliness De-Biasing passage did not 
alleviate negative mood. First, there may have been a floor effect in baseline levels of negative mood, which would have prevented significant decreases in negative mood in response to the manipulation. ${ }^{4}$ Perhaps more likely, the Loneliness De-Biasing passage may not have enabled a reappraisal as intended; this is supported by the finding that this passage did not challenge individuals' beliefs about their time alone any more than did a control passage.

It is a strength of the current study that we included two experimental conditions (Solitude Benefits and Loneliness De-Biasing) in order to determine what type of manipulation more effectively enables a reappraisal. Taken together, our results suggest that to help people reap the benefits of solitude, it may be more useful to reframe time alone as positive (i.e., solitude) rather than directly address the stigma associated with loneliness.

\section{Hypotheses III and IV: Trait Loneliness and Compulsive Social Media Use as Moderators of Mood Change}

The effect of the manipulation on changes in mood was not moderated by either trait loneliness or compulsive social media use. Thus, our Hypotheses III and IV were not supported. However, these findings are encouraging, as they suggest that anyone-regardless of loneliness levels or social-media related behaviors-may benefit from changing how they think about their time alone.

\section{Hypothesis V: Trait Reappraisal as a Moderator of Mood Change}

Trait reappraisal significantly moderated the relationship between condition and changes in positive mood, but not in the direction we had initially predicted. We found that within the Loneliness De-Biasing condition, individuals low in trait reappraisal experienced smaller reductions in positive mood than did those high in trait reappraisal. In other words, reading about the high prevalence of loneliness buffered against a dramatic drop in positive mood, but only for individuals who do not routinely use cognitive reappraisal to regulate their emotions.

\footnotetext{
${ }^{4}$ The distribution of baseline levels of negative mood exhibited a strong right skew, suggesting a floor effect. We conducted post-hoc exploratory analyses to examine a subsample of participants in the highest quartile of baseline negative mood. In this subsample, negative mood significantly decreased across conditions, as determined through a series of independent samples $t$-tests. Negative mood decreased more in this subsample $\left(M_{\text {Difference }}=3.54\right)$ than it did in the full sample $\left(M_{\text {Difference }}=1.17\right)$. However, the reappraisal conditions did not effect a larger decrease in negative mood than did the control condition, according to an exploratory ANOVA. It is important to note that our power was significantly attenuated in this analysis.
}

Previous research suggests that greater trait reappraisal predicts reduced affective reactivity to stressful events (Gross and John 2003). Surprisingly, our study showed that lower, rather than higher, levels of trait reappraisal predicted decreased emotional reactivity (i.e., a less dramatic drop in positive mood) after a reappraisal manipulation. There are a few possible explanations for these results. First, perhaps individuals who do not use reappraisal often are more impacted by reading information that contradicts their beliefs and, consequently, have a greater emotional response to that information. Second, individuals who routinely use reappraisal may have been more likely to already view loneliness as common. Nonetheless, our findings are encouraging - they suggest that individuals who do not regularly use reappraisal, rather than being placed at a disadvantage for reappraisal-based interventions, would indeed reap the greatest benefits.

\section{Limitations}

Our study is not without limitations. First and foremost, none of our hypotheses was fully supported. Nevertheless, this study makes an important contribution in how we might conceptualize the reframing of our time alone. Rather than aiming to increase positive affect, perhaps we should focus on conserving it.

Second, participants were not given the choice to sit alone but were instead instructed to do so. Previous research has considered the role of personal choice in our appraisals of our time alone. While solitude typically implies the deliberate choice to be alone, loneliness (frequently described as 'unwilling solitude') occurs when we feel alone against our wishes (Long and Averill 2003; Nguyen et al. 2018). Instructing individuals to sit alone may have negatively impacted their mood, and the effect of the manipulation may have differed for those who wanted to be alone versus those who did not. However, there is some ecological validity to our methodology, as we all experience times where we must be alone even if we do not want to be (Nance and Mays 2013). In addition, the Solitude Benefits passage may have produced a demand effect by prompting participants to report more positive experience of time alone; the fact that the purpose of our experiment was masked increases our confidence that such demand effects were minimized.

A third limitation is that the PANAS may not have adequately captured emotions of interest. Research to date has observed that solitude differentially affects high-arousal and low-arousal mood; solitude is linked to decreased higharousal positive affect but increased low-arousal positive affect (Pauly et al. 2017). Since the PANAS contains predominantly high-arousal emotions, total scores may not have been sensitive to changes in low-arousal emotions (Kuppens 
et al. 2013). Future studies examining the effects of solitude on our affective state may seek to (1) measure low-arousal positive mood states (e.g. calmness, thoughtfulness) that are more theoretically relevant to solitude and (2) assess changes in high- and low-arousal mood separately.

Finally, it is unknown whether reductions in positive mood while sitting alone for a brief period were caused by processes commensurate with those of chronic loneliness. For example, participants may have simply experienced boredom while sitting alone for only $10 \mathrm{~min}$ (Hsee et al. 2010), as opposed to the sadness, despair, and other emotional pains associated with long-term periods of isolation. That said, even brief and involuntary moments of time alone may be relevant to the study of loneliness. Previous studies have shown that both momentary and stable feelings of loneliness produce increased cortisol levels (Doane and Adam 2010). Further, experience sampling method (ESM) studies have shown that momentary feelings of loneliness predict future loneliness (Mote et al. 2020). Thus, our appraisals of brief moments of time alone, such as those examined in the current study, may influence more stable and long-term feelings of loneliness (Troy et al. 2010).

\section{Future Directions}

This examination has sparked several questions that should be addressed by further research. For instance, do our results generalize to a sample of lonelier individuals? The manipulation may have differentially impacted lonely vs. nonlonely individuals: those who already valued their alone time may not have derived as much benefit from the manipulation, whereas those who consistently feel lonely when alone may have been powerfully impacted by the idea that solitude and loneliness are distinct experiences. This possibility indicates a replication of the current study with a sample of individuals high in trait loneliness.

Second, do our findings generalize to older populations? As our sample size was skewed toward young adults, our results may not reflect the experiences of middle-aged and older individuals. Large-scale surveys have shown striking differences among generational cohorts in quantity of time spent alone; Americans aged 40 or younger spend approximately 3.5 waking hours alone daily, while those over aged 60 or older spend over 7 waking hours alone (Livingston 2019). Despite these differences, however, young adults are most affected by loneliness (Cigna 2018; Williams and Braun 2019). Thus, there is reason to believe that attitudes toward alone time - and subsequent appraisals of that timemight evolve across the lifespan (Pauly et al. 2017). Ultimately, future research should aim to represent a broader range of age groups.
Finally, are there any noteworthy differences between individuals who sat alone and unplugged (i.e., followed directions in our experiment) versus those who turned to technology (e.g., browsed the internet on the laboratory computer) while sitting alone? As individuals who did not comply with experimental instructions were excluded from our analyses, our sample may have been somewhat selfselecting. In addition, these individuals who struggled to sit alone might have benefited most from our cognitive intervention. Examining meta-data such as these, or conducting experiments in a more naturalistic paradigm that allows for choice in the use of technology and other alone time activities, may allow greater insight into who benefits most from reappraisal training. One possibility is a longitudinal study that employs an ESM design to test the effect of reappraisal training on loneliness and time alone experiences across a span of age ranges. Such a methodology would yield more ecologically valid results than a single-session study such as ours.

\section{Clinical Implications}

Our study suggests that clinicians working with individuals suffering from loneliness may have recourse to more than just social skills interventions or motivating patients to increase social contact. As a transdiagnostic element of many different mental disorders, loneliness can also be treated with cognitive flexibility techniques such as those found in the Unified Protocol (UP; Barlow et al. 2011). In this framework, patients would identify automatic negative thoughts surrounding isolation, and challenge such thoughts with corrective information such as those found in our manipulations.

This possibility is especially encouraging given the current challenges that the COVID-19 pandemic poses. To prevent spread of the disease, governments across the globe have implemented stay at home orders and "social distancing" practices (Banerjee and Rai 2020). Due to reduced social interactions and physical confinement, millions of people are experiencing increased isolation (Fiorillo and Gorwood 2020). Understandably, researchers believe COVID-19 will exacerbate the global loneliness epidemic (Maheshwari and Bronsther 2020).

Regardless of COVID-19's eventual course, its mental health consequences, including increases in loneliness, will persist, and may develop into longer-term health problems (Fiorillo and Gorwood 2020). Individuals are encouraged to maintain contact with friends and family members by virtual means (Fiorillo and Gorwood 2020). However, limited opportunities for social contact call for novel approaches to reducing loneliness (Banerjee and Rai 2020). 
In considering a path forward, Banerjee and Rai (2020) propose that we must focus on transforming loneliness into solitude. Indeed, our study identifies cognitive reappraisal as a potential mechanism to do exactly that. As cognitive reappraisal has been most useful when a situation cannot be changed or acted upon, it may be an effective tool for reframing time alone during COVID-19, when in-person social contact is no longer an option (Gross and John, 2003). We must focus on what we, ourselves, can do to alleviate feelings of loneliness (Banerjee and Rai 2020; Jeste et al. 2020): reappraising time alone may be one viable strategy to do so.

\section{Conclusion}

This study is the first to examine whether challenging maladaptive thoughts helps us experience a brief period of time alone in a more positive way. Our results provide preliminary evidence that cognitive reappraisal interventions effectively challenge negative beliefs about being alone and promote resilience to decrements in positive mood associated with alone time. Individuals who do not routinely exercise cognitive reappraisal in their daily lives may especially benefit from such an approach. However, the efficacy of cognitive reappraisal interventions should be determined by further replication of this work, especially in diverse populations, including those suffering from greater psychopathological severity.

Acknowledgements We thank Nicole Iannella, Silvana Gomez, and William Ryan for their assistance in testing participants. This article is based on a Senior Honors Thesis project in the Department of Psychology at Harvard University conducted by the first author and cosupervised by the second and third authors.

Author Contributions MR developed the study concept. All authors contributed to the study design. Data collection was performed by MR. $\mathrm{MR}$ and BWB conducted the data analysis and interpretation under the supervision of RJM. MR drafted the paper, and BWB and RJM provided critical revisions. All authors approved the final version of the manuscript for submission.

Funding This study was funded by a grant from the Harvard College Research Program awarded to the first author.

Data/Code Availability The dataset analyzed in the current study, as well as the RStudio code used in all analyses, is available in the Open Science Forum repository: osf.io/wsv3y.

\section{Compliance with Ethical Standards}

Conflict of Interest Micaela Rodriguez, Benjamin W. Bellet and Richard J. McNally declare that they have no conflict of interest.

Ethical Approval All procedures performed in this study involving human participants were in accordance with the ethical standards of the institutional committee and with the 1964 Helsinki declaration and its later amendments or comparable ethical standards. This study was performed in line with the principles of the Declaration of Helsinki. Approval was granted by the Committee on the Use of Human Subjects of Harvard University (Date: May 23, 2019/Protocol No. 19-0712).

Animal Rights No animal studies were carried out by the authors for this article.

Informed Consent Informed consent was obtained from all individual participants in this study.

\section{References}

Aalbers, G., McNally, R. J., Heeren, A., de Wit, S., \& Fried, E. I. (2019). Social media and depression symptoms: A network perspective. Journal of Experimental Psychology: General, 148, 1454-1462. https://doi.org/10.1037/xge0000528.

Allan, S., \& Gilbert, P. (1995). A social comparison scale: Psychometric properties and relationship to psychopathology. Personality and Individual Differences, 19, 293-299. https://doi. org/10.1016/0191-8869(95)00086-L.

Banerjee, D., \& Rai, M. (2020). Social isolation in Covid-19: The impact of loneliness. International Journal of Social Psychiatry. https://doi.org/10.1177/0020764020922269.

Barlow, D. H., Farchione, T. J., Fairholme, C. P., Ellard, K. K., Boisseau, C. L., Allen, L. B., et al. (2011). Treatments that work. Unified protocol for transdiagnostic treatment of emotional disorders: Therapist guide. Oxford: Oxford University Press.

Ben-Ari, A. (2012). Rethinking closeness and distance in intimate relationships: Are they really two opposites? Journal of Family Issues, 33, 391-412. https://doi.org/10.1177/0192513X11 415357.

Bessaha, M. L., Sabbath, E. L., Morris, Z., Malik, S., Scheinfeld, L., \& Saragossi, J. (2020). A systematic review of loneliness interventions among non-elderly adults. Clinical Social Work Journal, 48, 110-125. https://doi.org/10.1007/s10615-019-00724-0.

Boss, L., Kang, D.-H., \& Branson, S. (2015). Loneliness and cognitive function in the older adult: A systematic review. International Psychogeriatrics, 27, 541-553. https://doi.org/10.1017/S1041 610214002749

Brooks, A. C. (2018). An epidemic of loneliness in America? New York Times. Retrieved December 8, 2018, from https://www.nytim es.com/2018/12/08/opinion/letters/loneliness-epidemic.html.

Cacioppo, S., Grippo, A. J., London, S., Goossens, L., \& Cacioppo, J. T. (2015). Loneliness: Clinical import and interventions. Perspectives on Psychological Science, 10, 238-249. https://doi. org/10.1177/1745691615570616.

Cacioppo, J. T., Hughes, M. E., Waite, L. J., Hawkley, L. C., \& Thisted, R. A. (2006). Loneliness as a specific risk factor for depressive symptoms: Cross-sectional and longitudinal analyses. Psychology and Aging, 21, 140-151. https://doi. org/10.1037/0882-7974.21.1.140.

Cacioppo, J. T., \& Patrick, W. (2008). Loneliness: Human nature and the need for social connection. New York: W. W. Norton \& Co.

Cain, S. (2012). The rise of the new groupthink. New York Times. Retrieved January 13, 2012 from https://www.nytim es.com/2012/01/15/opinion/sunday/the-rise-of-the-new-group think.html.

Cigna. (2018). Cigna U.S. loneliness index. Survey of 20,000 Americans examining behaviors driving loneliness in the United States. Retrieved June 29, 2020, from https://www.cigna.com/newsroom/ news-releases/2018/new-cigna-study-reveals-loneliness-at-epide mic-levels-in-america 
Cornwell, E. Y., \& Waite, L. J. (2009). Social disconnectedness, perceived isolation, and health among older adults. Journal of Health and Social Behavior, 50, 31-48. https://doi.org/10.1177/00221 4650905000103.

Deri, S., Davidai, S., \& Gilovich, T. (2017). Home alone: Why people believe others' social lives are richer than their own. Journal of Personality and Social Psychology, 113, 858-877. https://doi. org/10.1037/pspa0000105.

Dill, J. C., \& Anderson, C. A. (1999). Loneliness, shyness, and depression: The etiology and interrelationships of everyday living. In T. Joiner \& J. C. Coyne (Eds.), The interactional nature of depression: Advances in interpersonal approaches (pp. 93-125). Washington D.C: American Psychological Association. https://doi. org/10.1037/10311-004.

Doane, L. D., \& Adam, E. K. (2010). Loneliness and cortisol: Momentary, day-to-day, and trait associations. Psychoneuroendocrinology, 35, 430-441. https://doi.org/10.1016/j.psyne uen.2009.08.005.

Fiorillo, A., \& Gorwood, P. (2020). The consequences of the COVID19 pandemic on mental health and implications for clinical practice. European Psychiatry, 63, e32. https://doi.org/10.1192/j.eurps y.2020.35

Gross, J. J., \& John, O. P. (2003). Individual differences in two emotion regulation processes: Implications for affect, relationships, and well-being. Journal of Personality and Social Psychology, 85, 348-362. https://doi.org/10.1037/0022-3514.85.2.348.

Hawkley, L. C., \& Cacioppo, J. T. (2010). Loneliness matters: A theoretical and empirical review of consequences and mechanisms. Annals of Behavioral Medicine, 40, 218-227. https://doi. org/10.1007/s12160-010-9210-8.

Hawkley, L. C., Masi, C. M., Berry, J. D., \& Cacioppo, J. T. (2006). Loneliness is a unique predictor of age-related differences in systolic blood pressure. Psychology and Aging, 21, 152-164. https:// doi.org/10.1037/0882-7974.21.1.152.

Heinrich, L. M., \& Gullone, E. (2006). The clinical significance of loneliness: A literature review. Clinical Psychology Review, 26, 695-718. https://doi.org/10.1016/j.cpr.2006.04.002.

Holt-Lunstad, J. (2017). The potential public health relevance of social isolation and loneliness: Prevalence, epidemiology, and risk factors. Public Policy \& Aging Report, 27, 127-130. https://doi. org/10.1093/ppar/prx030.

Holwerda, T. J., Deeg, D. J. H., Beekman, A. T. F., van Tilburg, T. G., Stek, M. L., Jonker, C., et al. (2014). Feelings of loneliness, but not social isolation, predict dementia onset: Results from the Amsterdam Study of the Elderly (AMSTEL). Journal of Neurology, Neurosurgery, and Psychiatry, 85, 135-142. https://doi. org/10.1136/jnnp-2012-302755.

Hsee, C. K., Yang, A. X., \& Wang, L. (2010). Idleness aversion and the need for busyness. Psychological Science, 21, 926-930. https ://doi.org/10.1177/0956797610374738.

Jamieson, J. P., Mendes, W. B., Blackstock, E., \& Schmader, T. (2010). Turning the knots in your stomach into bows: Reappraising arousal improves performance on the GRE. Journal of Experimental Social Psychology, 46, 208-212. https://doi.org/10.1016/j. jesp.2009.08.015.

Jeste, D. V., Lee, E. E., \& Cacioppo, S. (2020). Battling the modern behavioral epidemic of loneliness: Suggestions for research and interventions. JAMA Psychiatry, 77, 553-554. https://doi. org/10.1001/jamapsychiatry.2020.0027.

Jordan, A. H., Monin, B., Dweck, C. S., Lovett, B. J., John, O. P., \& Gross, J. J. (2011). Misery has more company than people think: Underestimating the prevalence of others' negative emotions. Personality \& Social Psychology Bulletin, 37, 120-135. https://doi. org/10.1177/0146167210390822.

Kross, E., Verduyn, P., Demiralp, E., Park, J., Lee, D. S., Lin, N., et al. (2013). Facebook use predicts declines in subjective well-being in young adults. PLoS ONE, 8(8), e69841. https://doi.org/10.1371/ journal.pone.0069841.

Kuppens, P., Tuerlinckx, F., Russell, J. A., \& Barrett, L. F. (2013). The relation between valence and arousal in subjective experience. Psychological Bulletin, 139, 917-940. https://doi.org/10.1037/ a0030811.

Larson, R. W. (1997). The emergence of solitude as a constructive domain of experience in early adolescence. Child Development, 68, 80-93. https://doi.org/10.2307/1131927.

Larson, R., Csikszentmihalyi, M., \& Graef, R. (1982). Time alone in daily experience: Loneliness or renewal. Loneliness: A sourcebook of current theory, research and therapy (pp. 40-53). New York: Wiley Publishers.

Larson, R., \& Lee, M. (1996). The capacity to be alone as a stress buffer. Journal of Social Psychology, 136, 5-16. https://doi. org/10.1080/00224545.1996.9923024.

Lau, S., \& Gruen, G. E. (1992). The social stigma of loneliness: Effect of target person's and perceiver's sex. Personality and Social Psychology Bulletin, 18, 182-189. https://doi. org/10.1177/0146167292182009.

Leary, M. R., Herbst, K. C., \& McCrary, F. (2003). Finding pleasure in solitary activities: Desire for aloneness or disinterest in social contact? Personality and Individual Differences, 35, 59-68. https://doi.org/10.1016/S0191-8869(02)00141-1.

Lim, M. H., Rodebaugh, T. L., Zyphur, M. J., \& Gleeson, J. F. (2016). Loneliness over time: The crucial role of social anxiety. Journal of Abnormal Psychology, 125, 620-630.

Liu, Q. Q., Zhou, Z. K., Yang, X. J., Niu, G. F., Tian, Y., \& Fan, C. Y. (2017). Upward social comparison on social network sites and depressive symptoms: A moderated mediation model of selfesteem and optimism. Personality and Individual Differences, 113, 223-228. https://doi.org/10.1016/j.paid.2017.03.037.

Livingston, G. (2019). On average, older adults spend over half their waking hours alone. Retrieved March 18, 2020, from Pew Research Center website: https://pewrsr.ch/2xqKeW6.

Long, C. R., \& Averill, J. R. (2003). Solitude: An exploration of benefits of being alone. Journal for the Theory of Social Behaviour, 33, 21-44. https://doi.org/10.1111/1468-5914.00204.

Luo, Y., Hawkley, L. C., Waite, L. J., \& Cacioppo, J. T. (2012). Loneliness, health, and mortality in old age: A national longitudinal study. Social Science \& Medicine, 74, 907-914. https:// doi.org/10.1016/j.socscimed.2011.11.028.

Madden, M., \& Rainie, L. (2015). Americans' attitudes about privacy, security and surveillance. Retrieved June 22, 2020, from Pew Research Center: Internet, Science \& Technology website: https://www.pewresearch.org/internet/2015/05/20/ameri cans-attitudes-about-privacy-security-and-surveillance/.

Maheshwari, S., \& Bronsther, R. (2020). COVID-19 in the era of loneliness. Current Psychiatry, 19, 31-33.

Masi, C. M., Chen, H.-Y., Hawkley, L. C., \& Cacioppo, J. T. (2011). A meta-analysis of interventions to reduce loneliness. Personality and Social Psychology Review, 15, 219-266. https://doi. org/10.1177/1088868310377394.

Meerkerk, G. J., Van Den Eijnden, R. J. J. M., Vermulst, A. A., \& Garretsen, H. F. L. (2009). The compulsive internet use scale (CIUS): Some psychometric properties. Cyberpsychology \& Behavior: The Impact of the Internet, Multimedia and Virtual Reality on Behavior and Society, 12, 1-6. https://doi. org/10.1089/cpb.2008.0181.

Mote, J., Gonzalez, R., Kircos, C., Gard, D. E., \& Fulford, D. (2020). I'm gonna make myself lonesome when you go: Predictors of loneliness in daily life (pre-print). PsyArXiv. Retrieved May 18, 2020, from https://doi.org/10.31234/osf.io/pr79g

Nance, W. Z., \& Mays, M. (2013). Exploring the role of time alone in modern culture. Paper presented at the American Counseling Association Conference, Cincinnati, $\mathrm{OH}$. 
Nguyen, T. T., Ryan, R. M., \& Deci, E. L. (2018). Solitude as an approach to affective self-regulation. Personality and Social Psychology Bulletin, 44, 92-106. https://doi.org/10.1177/0146167217 733073.

Pauly, T., Lay, J. C., Nater, U. M., Scott, S. B., \& Hoppmann, C. A. (2017). How we experience being alone: Age differences in affective and biological correlates of momentary solitude. Gerontology, 63, 55-66. https://doi.org/10.1159/000450608.

Rotenberg, K. J., \& MacKie, J. (1999). Stigmatization of social and intimacy loneliness. Psychological Reports, 84, 147-148. https ://doi.org/10.2466/pr0.1999.84.1.147.

Russell, D. W. (1996). UCLA Loneliness Scale (version 3): Reliability, validity, and factor structure. Journal of Personality Assessment, 66, 20-40. https://doi.org/10.1207/s15327752jpa6601_2.

Skues, J., Williams, B., Oldmeadow, J., \& Wise, L. (2016). The effects of boredom, loneliness, and distress tolerance on problem internet use among university students. International Journal of Mental Health and Addiction, 14, 167-180. https://doi.org/10.1007/s1146 9-015-9568-8.

Stravynski, A., \& Boyer, R. (2001). Loneliness in relation to suicide ideation and parasuicide: A population-wide study. Suicide \& LifeThreatening Behavior: Washington, 31, 32-40.

Tilvis, R. S., Kähönen-Väre, M. H., Jolkkonen, J., Valvanne, J., Pitkala, K. H., \& Strandberg, T. E. (2004). Predictors of cognitive decline and mortality of aged people over a 10-year period. Journals of Gerontology Series A: Biological Sciences and Medical Sciences, 59, M268-M274. https://doi.org/10.1093/gerona/59.3.M268.

Troy, A. S., Wilhelm, F. H., Shallcross, A. J., \& Mauss, I. B. (2010). Seeing the silver lining: Cognitive reappraisal ability moderates the relationship between stress and depressive symptoms. Emotion, 10, 783-795. https://doi.org/10.1037/a0020262.

Twenge, J. M., Martin, G. N., \& Spitzberg, B. H. (2019). Trends in U.S. Adolescents' media use, 1976-2016: The rise of digital media, the decline of TV, and the (near) demise of print. Psychology of Popular Media Culture, 8, 329-345. https://doi.org/10.1037/ ppm0000203.

Verduyn, P., Lee, D. S., Park, J., Shablack, H., Orvell, A., Bayer, J., et al. (2015). Passive Facebook usage undermines affective well-being: Experimental and longitudinal evidence. Journal of Experimental Psychology: General, 144, 480-488. https://doi. org/10.1037/xge0000057.

Verduyn, P., Ybarra, O., Résibois, M., Jonides, J., \& Kross, E. (2017). Do social network sites enhance or undermine subjective wellbeing? A critical review. Social Issues and Policy Review, 11, 274-302. https://doi.org/10.1111/sipr.12033.

Wang, J.-L., Wang, H.-Z., Gaskin, J., \& Hawk, S. (2017). The mediating roles of upward social comparison and self-esteem and the moderating role of social comparison orientation in the association between social networking site usage and subjective wellbeing. Frontiers in Psychology, 8, 1-9. https://doi.org/10.3389/ fpsyg.2017.00771.

Watson, D., Clark, L., \& Tellegen, A. (1988). Development and validation of brief measures of positive and negative affect: The PANAS scales. Journal of Personality and Social Psychology, 54, 1063 1070. https://doi.org/10.1037//0022-3514.54.6.1063.

Williams, S. E., \& Braun, B. (2019). Loneliness and social isolationA private problem, a public issue. Journal of Family \& Consumer Sciences, 111, 7-14. https://doi.org/10.14307/JFCS111.1.7.

Wilson, R. S., Krueger, K. R., Arnold, S. E., Schneider, J. A., Kelly, J. F., Barnes, L. L., et al. (2007). Loneliness and risk of Alzheimer disease. Archives of General Psychiatry, 64, 234-240. https://doi. org/10.1001/archpsyc.64.2.234.

Wilson, T. D., Reinhard, D. A., Westgate, E. C., Gilbert, D. T., Ellerbeck, N., Hahn, C., et al. (2014). Just think: The challenges of the disengaged mind. Science, 345, 75-77. https://doi.org/10.1126/ science. 1250830.

Yang, C.-C. (2016). Instagram use, loneliness, and social comparison orientation: Interact and browse on social media, but don't compare. Cyberpsychology, Behavior \& Social Networking, 19, 703-708. https://doi.org/10.1089/cyber.2016.0201.

Publisher's Note Springer Nature remains neutral with regard to jurisdictional claims in published maps and institutional affiliations. 